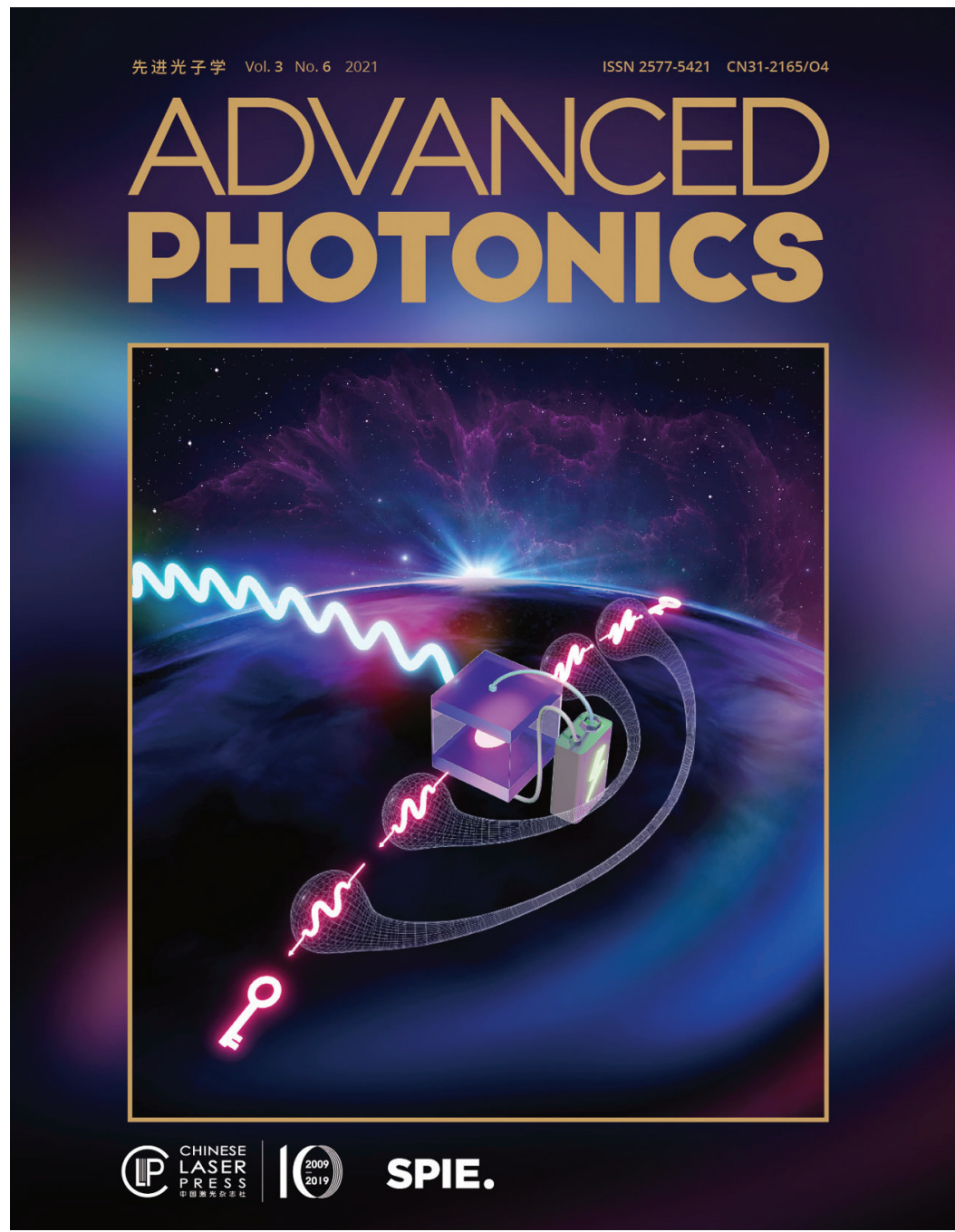

\title{
About the cover: Advanced Photonics Volume 3, Issue 6
}

The image on the cover for Advanced Photonics Volume 3 Issue 6 depicts the generation of polarization-entangled photon pairs by an optically excited semiconductor quantum dot. The dot is placed in a $\mathrm{p}-\mathrm{i}-\mathrm{n}$ diode structure, supplied with a forward-bias voltage, to control its internal charge configuration and to allow the generation of an uninterrupted stream of photon pairs. These photon pairs are used to perform quantum key distribution between two parties, located in different buildings, connected via a 350-metre-long optical fiber. The generated keys were used to encrypt and decrypt a message in a theoretically informationsecure manner.

The image is based on original research presented in the report by Christian Schimpf, Santanu Manna, et al., "Entanglement-based quantum key distribution with a blinkingfree quantum dot operated at a temperature up to $20 \mathrm{~K}$," Adv. Photonics 3(6), 065001 (2021). 